\title{
Amadeus
}

International Multidisciplinary Journal IISSN 2525-3281

DOI: 10.14295 /aimj.v4i7.95

\section{The use of Ursodesoxicolic Acid in Prevention of \\ Collelitiase After Bariatric Surgery: \\ A Systematic Review of Literature}

Bráulio Filgueira Magalhães ${ }^{1}$; Pedro de Sousa Leite ${ }^{2}$, Gabriella Rocha Moreira ${ }^{3}$; Yanny Brena Alencar Araújo ${ }^{4}$; Natália Mendes Cruz; Francisco Felippe de Araújo Rolim $^{6}$
Abstract: Morbid obesity is considered a serious public health problem generating high human and financial costs. The situation that promotes the highest morbidity after bariatric surgery is the development of cholelithiasis, especially in the first 6 months. Given this process, ursodeoxycholic acid is increasingly being used in the postoperative period due to a reduction in the rate of cholelithiasis. However, there are discussions of which prophylactic method to use. In this context, a systematic review was performed to evaluate the efficacy and use of ursodeoxycholic acid in preventing cholelithiasis after bariatric surgery. After methodological analysis, 6 articles were selected. These studies suggest that administration of 500 to $1000 \mathrm{mg}$ of UDCA for a period of 6 months, depending on the technique used, is significantly associated with decreased incidence of cholelithiasis, with good adherence, costeffectiveness and few side effects.

Keywords: Ursodeoxycholic Acid, Cholelithiasis, Bariatric Surgery.

\footnotetext{
${ }^{1}$ Resident General Surgery- Juazeiro do Norte Medical School (FMJ), Juazeiro do Norte-CE, Brazil, braulio.magalhaes@hotmail.com.

${ }^{2}$ Graduating in Medicine from the Estadio de Juazeiro do Norte Medical School. Juazeiro do Norte, Ceará, Brazil; pedroed1913@hotmail.com ;.

${ }^{3}$ Medical Student, Estacio de Juazeiro do Norte Medical School. Juazeiro do Norte, Ceará, Brazil; gabs_rm@live.com

${ }^{4}$ Medical student, Estacio de Juazeiro do Norte Medical School. Juazeiro do Norte, Ceará, Brazil; yannybrena08@hotmail.com

${ }^{5}$ Medical Student, Estacio de Juazeiro do Norte Medical School. Juazeiro do Norte, Ceará, Brazil; natalia.cruz92@hotmail.com

${ }^{6}$ Professor MSc. Juazeiro do Norte Medical School (FMJ), Juazeiro do Norte-CE, Brazil, felippe.rolim1@gmail.com
} 


\section{O uso do Ácido Ursodesoxicolico na prevenção de Colelitiase após Cirurgia Bariátrica: Uma Revisão Sistemática da Literatura}

\begin{abstract}
Resumo: A obesidade mórbida é considerada um grave problema de saúde pública gerando elevados custos humanos e financeiros. A situação que promove maior morbidade após realização da cirurgia bariátrica é o desenvolvimento do quadro de colelitíase, principalmente nos primeiros 6 meses. Diante desse processo, cada vez mais se faz uso do ácido ursodesoxicolico no período pós-operatório decorrente de proporcionar uma redução da taxa de colelitíase. Entretanto, existem discussões de qual método profilático utilizar. Neste contexto foi realizado uma revisão sistemática com o objetivo de avaliar a eficácia e uso do ácido ursodesoxicolico na prevenção do quadro de colelitíase após realização da cirurgia bariátrica. Após análise metodológica foram selecionados 6 artigos. Esses estudos sugerem que a administração de 500 a $1000 \mathrm{mg}$ de UDCA por um período de 6 meses, dependendo da técnica utilizada esta significativamente associada a diminuição da incidência de colelitíase, com boa adesão, custo-efetividade e poucos efeitos colaterais.
\end{abstract}

Palavras-chave: Ácido Ursodesoxicolico, Colelitiase, Cirurgia Bariátric.

\section{Introdução}

A obesidade mórbida é considerada um grave problema de saúde pública gerando elevados custos humanos e financeiros. Nos Estados Unidos é descrita como uma das doenças com maior taxa de mortalidade no mundo. Diante desse cenário, a necessidade da realização de procedimentos cirúrgicos tornou-se ainda mais frequente, apresentando dados anuais nos Estados Unidos de 140.000. Quando se avalia o Brasil, foi detectado que 38,8 milhões de brasileiros com idade superior a 20 anos apresentavam sobrepeso e $11 \%$ eram considerados obesos (Machado et al., 2019).

De acordo com a Sociedade Brasileira de Cirurgia Bariátrica e Metabólica (SBCBM) (2018) o número de cirurgias bariátricas que foram realizadas no Brasil entre os anos de 2012 e 2017 sofreu um aumento de 46,7\%. Vale salientar que entre 2008 e 2017, o número de procedimentos cirúrgicos realizados pelo Sistema Único de Saúde (SUS) retratou um crescimento de $215 \%$.

O excesso de peso promove um aumento de diversos problemas de saúde, destacando disfunções cardíacas, hipertensão arterial, diabetes tipo II, osteoartrite como 
também alguns tipos de câncer que estão relacionados ao quadro de obesidade. Diante desse contexto, a perda excessiva de peso em um período curto com o uso de dietas e principalmente procedimentos cirúrgicos promove diversas alterações no organismo, desde benéficas a maléficas. A situação que promove maior morbidade após procedimento cirúrgico é o desenvolvimento do quadro de colelitíase, principalmente nos primeiros 6 meses, a depende da técnica utilizada, sendo a de RYGB normalmente mais associada (Ferrari, 2014).

Abdallah e outros colaboradores (2017) descreveram que o quadro de colelitíase pós-operatória (LC) é considerada como uma complicação latente da cirurgia bariátrica, no qual $45 \%$ dos pacientes que desenvolverem a obesidade mórbida iram desenvolvê-la. Esse processo ocorre devido à elevação do índice de massa corporal ser considerada como fator primordial para formação de cálculos biliares, principalmente no sexo feminino.

De acordo com Nabil, Khalil e Gamal (2019) a perda de pesa excessiva é identificada como principal fator de risco para o desenvolvimento do quadro de CL, apresentando uma morbidade de $35-38 \%$ dos pacientes após a realização da cirurgia bariátrica.

Abdallah e outros colaboradores (2017) relatam que a técnica de RYGB que por ser considerado um procedimento de má absorção, a incidência de CL é maior devido ocorrer alterações na composição da bile, principalmente o aumento da concentração do cálcio e da mucina, promovendo a formação de um bile supersaturada ou litogênica que acaba cristalizando, se depositando e por fim formando cálculos biliares. Por outro lado, já a LSG é considerada um procedimento puramente restritivo e com isso não se sabe os reais fatores envolvidos na gênese de CL, alguns autores avaliam que a relação da sua formação pode ser decorrente de uma lesão do ramo háptico do nervo vago promovendo assim uma disfunção da motilidade da vesícula biliar e predispondo a formação da CL decorrente da estase biliar.

Neste cenário foi desenvolvida a colecistectomia profilática com objetivo de prevenir a LC após cirurgia bariátrica, entretanto, apresenta dificuldades técnicas, como também proporciona a formação de complicações, prolonga o tempo operatório e a permanência hospitalar. Diante desse processo, cada vez mais se faz uso do ácido ursodesoxicólico (UDCA) no período pós-operatório decorrente de proporcionar uma redução do quadro de LC (Abdallah et al., 2017). 
Coupaye e outros colaboradores (2017) abordam em seu estudo que o ácido ursodesoxicólico (UDCA) é definido como um ácido biliar secundário que atua reduzindo o processo de saturação de colesterol da bile, como por exemplo em pacientes com diagnóstico de obesidade.

A realização da cirurgia bariátrica é considerada como tratamento ideal do quadro de obesidade mórbida, entretanto está alinhada ao risco de desenvolver o quadro de CL e com isso surgindo discursões a respeito de qual medida profilática ser utilizada. Sendo assim o uso do UDCA esta associado a impacto positivo como medida profilática do quadro de cálculo biliar no qual apresenta boa adesão, segurança e eficácia, no entanto é notório que na prática clínica existe diferenças em qual abordagem utilizada, no qual alguns autores recomendam a prescrição de forma preventiva com o UDCA, outros adotam a realização da colecistectomia profilática, enquanto que outros argumentam a não realização de nenhuma medida profilática decorrente da baixa incidência de CL após a cirurgia bariátrica. Nesse contexto, esta revisão sistemática busca avaliar a eficácia e os benefícios do uso do UDCA na prevenção do quadro de colelitíase após realização da cirurgia bariátrica.

\section{Materiais e Métodos}

A revisão sistemática apresenta como objetivo primordial realizar um processo de síntese das informações sobre o tema abordado com qualidade metodológica, com o propósito de evitar vieses em todas as etapas da pesquisa. Para tal, foram utilizados " $a$ priori" no protocolo do estudo, as seguintes etapas:
a) definição da pergunta de revisão;
b) critérios de inclusão e exclusão;
c) estratégias para a busca dos estudos;
d) seleção do material;
e) análise crítica dos estudos;
e) coleta e síntese dos dados.

\section{Definição da pergunta}


O uso do ácido ursodesoxicolico apresenta eficácia como medida de prevenção do quadro de colelitíase após realização da cirurgia bariátrica?

\section{Critérios de inclusão}

Consideraram-se elegíveis os estudos randomizados, prospectivos e retrospectivos publicados no período de janeiro de 2014 a novembro de 2019; em qualquer idioma e em revistas com revisão por pares, com fator de impacto e disponibilizados na íntegra. Além disso, os estudos deveriam descrever resultados de abordagens realizados em seres humanos e apresentassem uma amostra superior a 15 pacientes.

Foram incluídos todos os estudos que avaliaram a eficácia e os benéficos do UDCA como medida profilática do quadro de colelitíase após realização da cirurgia bariátrica. Artigos que aparecerem em mais de um banco de dados serão incluídos apenas uma vez, com prioridade à base de dados SCOPUS.

\section{Critérios de exclusão}

Foram excluídos relatos de caso, revisões, prefácios, comunicações breves e cartas ao editor, estudos com duplicata e/ou que apresentava erros sistemáticos, ambiguidade ou apresentação insuficiente dos resultados.

\section{Estratégia de busca e seleção do material}

Os estudos foram selecionados por meio de busca eletrônica nas bases de dados Medical Literature Analysis and Retrieval System Online (MEDLINE/PubMed) e Biblioteca Virtual em Saúde (BVS). Foi utilizado os descritores em DeCS Ácido Ursodesoxicólico, Cirurgia Bariátrica e Colelitíase e os MeSH terms Ursodeoxycholic Acid, bariatric surgery e Cholelithiasis utilizando o operador Booleando AND com os descritores combinados dois a dois.

Os estudos potencialmente relevantes para serem incluídos na revisão sistemática foram obtidos pela leitura dos títulos, resumos e do texto integral sempre que o resumo não fosse esclarecedor relativamente aos critérios utilizados para a decisão de inclusão ou 
exclusão. Um só investigador foi responsável pela extração dos seguintes dados: nome dos autores, título do artigo e ano de publicação; tipo do estudo; tamanho da amostra estudada, país do estudo; periódico; duração do uso do UDCA após cirurgia bariátrica e desfecho.

Foi realizada de forma ativa a busca manual (handsearching) por literatura cinzenta (relatórios e/ou documentos governamentais, dissertações e/ou teses, revisões bibliográficas, anais científicos, livros com pertinência teórica na área), além da utilização de artigos de revisão não elegíveis segundo os critérios de busca para o estudo, a fim de serem referenciados no trabalho para se realizar um embasamento teórico sobre o tema.

\section{Resultados}

Na fase de identificação dos artigos, obtiveram-se 1941 registros, dos quais 526 foram excluídos por serem duplicatas. Além disso, 602 foram excluídos após a análise dos critérios de inclusão e 106 por fugirem do tema, serem inconclusivos, com baixo teor metodológico e/ou por não responderem aos objetivos do estudo. Através da leitura detalhada dos estudos e pela confirmação de elegibilidade, 6 artigos foram inclusos como evidências. O tipo do estudo foi retrospectivo em dois estudos, prospectivo em quatro e prospectivo e randomizado em um estudo. Os estudos incluídos foram realizados no Egito (dois artigos), Estados Unidos da América, França, Alemanha e Brasil um estudo cada.

Foi elaborada a Tabela 1 no qual descreve as características dos estudos que foram finalmente incluídos na revisão sistemática.

Tabela 1: Características dos estudos que foram finalmente incluídos na revisão sistemática

\begin{tabular}{|c|c|c|c|c|c|c|c|}
\hline \multirow{2}{*}{$\begin{array}{c}\text { Autor/ } \\
\text { Ano }\end{array}$} & \multirow{2}{*}{$\begin{array}{l}\text { País de } \\
\text { Origem }\end{array}$} & \multirow{2}{*}{$\begin{array}{c}\text { Período do } \\
\text { estudo }\end{array}$} & \multirow{2}{*}{ Periódico } & \multirow{2}{*}{$\begin{array}{l}\text { Tipo de } \\
\text { Estudo }\end{array}$} & \multicolumn{2}{|c|}{$\begin{array}{c}\text { Tamanho da } \\
\text { amostra } \\
\text { (n) }\end{array}$} & \multirow{2}{*}{$\begin{array}{c}\text { Duração do } \\
\text { uso do } \\
\text { UDCA } \\
\text { Após } \\
\text { cirurgia } \\
\text { bariátrica }\end{array}$} \\
\hline & & & & & UDCA & Controle & \\
\hline $\begin{array}{c}\text { Abdallah } \\
\text { et al., } \\
2017\end{array}$ & Egito & $\begin{array}{c}\text { Janeiro de } \\
2010 \text { a Maio } \\
\text { de } 2015 .\end{array}$ & $\begin{array}{c}\text { Surgery } \\
\text { today }\end{array}$ & Retrospectivo & 247 & 159 & 6 meses \\
\hline $\begin{array}{l}\text { Adams et } \\
\text { al., } 2019\end{array}$ & $\begin{array}{l}\text { Estados } \\
\text { Unidos da } \\
\text { América }\end{array}$ & $\begin{array}{c}\text { Dezembro } \\
\text { de } 2011 \text { a } \\
\text { Abril de } \\
2013 .\end{array}$ & $\begin{array}{l}\text { Obesity } \\
\text { surgery }\end{array}$ & $\begin{array}{l}\text { Randomizado } \\
\text { e Prospectivo }\end{array}$ & 37 & 38 & 6 meses \\
\hline $\begin{array}{c}\text { Coupaye } \\
\text { et al., } \\
2017\end{array}$ & França & $\begin{array}{c}\text { Janeiro de } \\
2008 \text { a } 2015 \\
\text { de fevereiro. }\end{array}$ & $\begin{array}{c}\text { Surgery for } \\
\text { Obesity and } \\
\text { Related }\end{array}$ & Prospectivo & 228 & 168 & 6 meses \\
\hline
\end{tabular}




\begin{tabular}{|c|c|c|c|c|c|c|c|}
\hline & & & Diseases & & & & \\
\hline $\begin{array}{c}\text { Della } \\
\text { Penna et } \\
\text { al., } 2019\end{array}$ & Alemanha & $\begin{array}{c}\text { Janeiro de } \\
2007 \mathrm{e} \\
\text { Outubro de } \\
2017 . \\
\end{array}$ & $\begin{array}{l}\text { Obesity } \\
\text { surgery }\end{array}$ & Retrospectivo & 61 & 0 & 6 meses \\
\hline $\begin{array}{c}\text { Machado } \\
\text { et al., } \\
2019\end{array}$ & Brasil & $\begin{array}{c}\text { Janeiro de } \\
2014 \text { a } \\
\text { Dezembro } \\
\text { de } 2015 .\end{array}$ & $\begin{array}{c}\text { Acta Cir } \\
\text { Bras }\end{array}$ & Prospectivo & 69 & 68 & 12 meses \\
\hline $\begin{array}{l}\text { Nabil, } \\
\text { Khalil e } \\
\text { Gamal } \\
(2019)\end{array}$ & Egito & $\begin{array}{c}\text { Julho de } \\
2015 \text { e } \\
\text { Março de } \\
2018 .\end{array}$ & $\begin{array}{c}\text { Surgery for } \\
\text { Obesity and } \\
\text { Related } \\
\text { Diseases }\end{array}$ & Prospectivo & 100 & 100 & 6 meses \\
\hline
\end{tabular}

Fonte: elaborada pelos autores.

A Figura 1 demonstra o Fluxograma da busca dos estudos da revisão sistemática.

Figura 1: Fluxograma da busca dos estudos.

Registros identificados através de pesquisa de banco de dados $(n=1241$ (PUBMED) )
Registros adicionais identificados através de outras fontes $(n=700(B V S))$

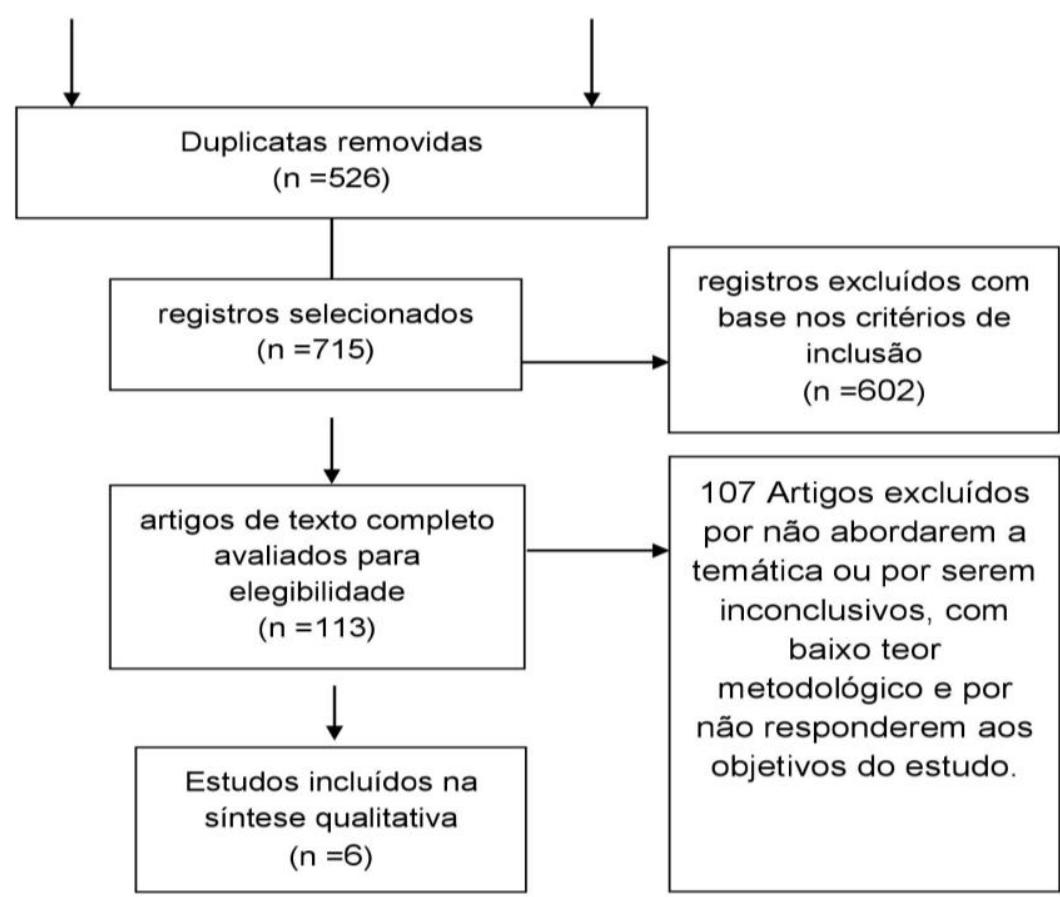


Fonte: Elaborado pelos autores

Na Tabela 2, a seguir, é possível identificar os principais resultados.

Tabela 2: Principais achados

\begin{tabular}{|c|c|c|}
\hline TÍTULO & OBJETIVO & DESFECHO \\
\hline $\begin{array}{l}\text { Role of } \\
\text { ursodeoxycholic acid } \\
\text { in the prevention of } \\
\text { gallstone formation } \\
\text { after laparoscopic } \\
\text { sleeve gastrectomy. }\end{array}$ & $\begin{array}{c}\text { Avaliar o papel do ácido } \\
\text { ursodeoxicólico (UDCA) } \\
\text { na prevenção de colelitíase } \\
\text { pós-operatória (CL) após } \\
\text { gastrectomia vertical } \\
\text { laparoscópica (LSG). }\end{array}$ & $\begin{array}{l}\text { Foi possível avaliar que o uso do UCDA } \\
\text { por um período de } 6 \text { meses após a } \\
\text { realização da cirurgia bariátrica em } \\
\text { pacientes com obesidade mórbida (IMC } \\
\text { basal médio pré-operatório de } 50,1 \pm 8,3 \\
\mathrm{~kg} / \mathrm{m} 2 \text { ) proporcionou uma redução } \\
\text { significativa (IC95\% } 0,001-018 ; \mathrm{P} \\
<0,0001 \text { ) nos primeiros } 12 \text { meses. No } \\
\text { qual, dos } 8 \text { pacientes que apresentaram o } \\
\text { quadro de CL todos estavam dentro do } \\
\text { grupo controle. }\end{array}$ \\
\hline $\begin{array}{l}\text { Randomized, } \\
\text { prospective } \\
\text { comparison of } \\
\text { ursodeoxycholic acid } \\
\text { for the prevention of } \\
\text { gallstones after } \\
\text { sleeve gastrectomy. }\end{array}$ & $\begin{array}{l}\text { Identificar a eficácia do } \\
\text { UDCA na prevenção de } \\
\text { cálculos biliares após a } \\
\text { gastrectomia vertical (SG). }\end{array}$ & $\begin{array}{l}\text { Concluiu-se que após a utilização de } 300 \\
\text { mg de UDCA duas vezes ao dia por } 6 \\
\text { meses após o SG identificou-se taxas } \\
\text { mais baixas de formação do quadro de } \\
\text { CL nos primeiros } 6 \text { meses com redução } \\
\text { estatisticamente significativa ( } \mathrm{p}= \\
\text { 0,032). Entretanto, sem diferença } \\
\text { significativa após } 1 \text { ano do } \\
\text { procedimento. Vale salientar que o } \\
\text { estudo apresenta algumas limitações, } \\
\text { entre elas, falha no acompanhamento } \\
\text { dos pacientes }(24 \%) \text { e um tamanho da } \\
\text { amostra pequena. }\end{array}$ \\
\hline $\begin{array}{l}\text { Evaluation of } \\
\text { incidence of } \\
\text { cholelithiasis after } \\
\text { bariatric surgery in } \\
\text { subjects treated or } \\
\text { not treated with } \\
\text { ursodeoxycholic } \\
\text { acid. }\end{array}$ & $\begin{array}{l}\text { Comparar a incidência } \\
\text { de CL entre pacientes } \\
\text { tratados ou não com UDCA } \\
\text { após RYGB e SG. }\end{array}$ & $\begin{array}{l}\text { Foi identificado que a dose de } 500 \\
\text { mg/dia é considerado mais eficiente para } \\
\text { prevenção do quadro de CL após } \\
\text { realização de SG do que após a RYGB } \\
\text { nos primeiros } 12 \text { meses ( } P=0,005 \text { ). } \\
\text { Entretanto, foi identificado um número } \\
\text { elevado de pacientes que não } \\
\text { continuaram o acompanhamento, com } \\
\text { isso, sendo necessária a realização de } \\
\text { mais estudos randomizados. }\end{array}$ \\
\hline
\end{tabular}




\begin{tabular}{|c|c|c|}
\hline $\begin{array}{l}\text { Ursodeoxycholic } \\
\text { Acid for } 6 \text { Months } \\
\text { After Bariatric } \\
\text { Surgery Is Impacting } \\
\text { Gallstone Associated } \\
\text { Morbidity in Patients } \\
\text { with Preoperative } \\
\text { Asymptomatic } \\
\text { Gallstones. }\end{array}$ & $\begin{array}{c}\text { Analisar retrospectivamente } \\
\text { o impacto da administração } \\
\text { UDCA profilático durante } 6 \\
\text { meses após a cirurgia } \\
\text { bariátrica sobre a } \\
\text { morbidade decorrente do } \\
\text { quadro de colelitíase } \\
\text { assintomática pré- } \\
\text { operatória. }\end{array}$ & $\begin{array}{l}\text { Foi identificado que } 96,8 \% \text { dos } \\
\text { pacientes que fizeram o tratamento com } \\
\text { o UDCA não desenvolveram sintomas } \\
\text { após o seu uso após procedimento } \\
\text { cirúrgico. Devido o estudo apresentar } \\
\text { como foco o uso do UDCA em pacientes } \\
\text { com colelitíase assintomática não foi } \\
\text { criado um grupo controle, apresentando } \\
\text { com isso uma limitação. }\end{array}$ \\
\hline $\begin{array}{l}\text { Ursodeoxycholic } \\
\text { acid in the } \\
\text { prevention of } \\
\text { gallstones in patients } \\
\text { subjected to Roux- } \\
\text { en-Y gastric bypass. }\end{array}$ & $\begin{array}{l}\text { Avaliar a contribuição do } \\
\text { ácido ursodeoxicólico } \\
\text { (UDCA) nos primeiros } 12 \\
\text { meses após o bypass } \\
\text { gástrico em Y de Roux na } \\
\text { prevenção da formação de } \\
\quad \text { cálculos biliares. }\end{array}$ & $\begin{array}{l}\text { Realizado um estudo retrospectivo com } \\
137 \text { pacientes, no qual } 69 \text { iniciaram } \\
\text { tratamento com UDCA, } 30 \text { dias após o } \\
\text { procedimento cirúrgico no qual foi } \\
\text { identificado que } 98,5 \% \text { não } \\
\text { desenvolveram colelitíase. Além disso, } \\
\text { foi possível avaliar que o grupo controle } \\
\text { apresentou a probabilidade de } 24,1 \\
\text { vezes mais chances de desenvolver } \\
\text { colelitíase (IC } 95 \%=3,1-189,4 \text {, p } \\
<0,001 \text { ). }\end{array}$ \\
\hline $\begin{array}{l}\text { Effect of oral } \\
\text { ursodeoxycholic acid } \\
\text { on cholelithiasis } \\
\text { following } \\
\text { laparoscopic sleeve } \\
\text { gastrectomy for } \\
\text { morbid obesity. }\end{array}$ & $\begin{array}{l}\text { Avaliar o efeito profilático } \\
\text { do UDCA na colelitíase } \\
\text { após LSG em pacientes } \\
\text { obesos mórbidos. }\end{array}$ & $\begin{array}{l}\text { Foi possível avaliar que o uso do UDCA } \\
\text { durante } 6 \text { meses pós cirurgia apresenta } \\
\text { resultados significativos na prevenção } \\
\text { do quadro de CL. Foi identificado que } \\
\text { apenas } 6 \% \text { dos pacientes que fizeram } \\
\text { uso da medicação, desenvolveram o } \\
\text { quadro de CL. }\end{array}$ \\
\hline
\end{tabular}

Fonte: elaborada pelos autores

\section{Discussão}

A obesidade é considerada como um fator de risco fortemente associada ao desenvolvimento de cálculos biliares, no qual após a realização de RYGB foi identificado uma prevalência de $10 \%$, como também $21 \%$ dos pacientes que apresentavam um quadro de colelitíase assintomática desenvolveram sintomas após o procedimento cirúrgico, sendo a perda excessiva de peso superior a 50\% como principal fator preditivo associado à realização da técnica (Della Penna et al., 2019).

O UDCA apresenta como benefícios proporcionar o bloqueio da evolução do quadro da doença hepática gordurosa não hepática para esteato-hepatite não alcoólica, decorrente da proteção gerada aos hepatócitos durante o manuseio de sais biliares em trauma mitocondrial. Além disso, é considerado como via de sinalização anti-apoptótica, 
função anti-inflamatória, antioxidante, imunomodulatória, propriedades anti-fibrótica (Machado et al., 2019).

Neste estudo foi identificado seis artigo que avaliaram o uso, a eficácias e as contribuições da administração do UDCA após a realização da cirurgia bariátrica, no qual foi possível avaliar que o seu uso pode ser considerado uma abordagem tolerada, viável com custo-benefício significativo, apresentando eficácia na redução da incidência pósoperatória de colelitíase, como também da formação dos sintomas em pacientes que apresentavam calculo biliar no período pré-operatório. Vale salientar que a dose com melhores resultados significativa dependeu de qual técnica utilizada, sendo que as identificadas com associação com o desenvolvimento do quadro de CL foi a RYGB e a $\mathrm{SG}$, com maior incidência de desenvolvimento a primeira. Além disso, é notório que pacientes que realizam necessitam de doses menores do UDCA como medida profilática do quadro de CL.

Adams e outros colaboradores (2019) realizaram um estudo randomizado no qual descrevem que a utilização do UDCA esta associado a uma incidência baixa dos casos de LC após a realização do SG, principalmente nos 6 primeiros meses de uso, sem predileção pelo sexo ou etnia, entretanto, afirma que existe a necessidade da realização de estudos que identifique uma diretriz padrão em relação a tempo e dose para que seu uso seja mais eficaz. Isso é de fundamental importância, já que é notório que o UDCA apresenta efeito de proteção para formação do quadro de cálculo biliar.

Machado e outros colaboradores (2019) realizaram um estudo prospectivo no município de Fortaleza-CE, no qual os pacientes fizeram uso do UDCA (300mg/dia) durante 12 meses, sendo que os autores afirmam que quando seu uso é iniciado durante a fase rápida de perda de peso apresenta eficácia significativa $(\mathrm{p}<0,001)$ na prevenção do quadro de colelitíase após realização de RYGB.

Já Coupaye e outros colaboradores (2017) descrevem que é notório o sua eficácia na diminuição de casos de colelitíase (CL) após a realização da técnica RYGB, sendo que a dose da prescrição de UDCA foi de $600 \mathrm{mg} /$ dia durante 6 meses pós cirurgia em pacientes com idade superior a $20^{\mathrm{a}}$ anos, de acordo com orientações da corrente americana e francesa com o objetivo de proporcionar a redução de formação dos cálculos biliares. Nos casos da utilização da técnica SG a estratégia preventiva também não se encontra definida, no qual a dose mais recomendada sobre o uso da UDCA é de 300mg/dia com identificação de resultados significativos para redução do quadro de CL nos primeiros 6 meses. 
Abdallah e outros colaboradores (2017) afirmam que as técnicas cirúrgicas que são utilizadas pra realização da cirurgia bariátrica em pacientes com quadro e obesidade mórbida predispõem de maneira significativa o desenvolvimento da LC, no qual a rápida perda de peso, a formação de bile litogênica com concentrações elevadas de colesterol e os mecanismos formados pelos procedimentos cirúrgicos são fatores cruciais para a formação dos cálculos biliares, além disso, promover a formação de sintomas e complicações em pacientes que tinha história prévia de colelitíase assintomática. Percebe-se também que, a incidência do quadro de LC está intimamente ligada às técnicas de circulação gástrica em Y roux (RYGB) e a gastrectomia vertical (SG), sendo que alguns estudos observacionais descrevem a primeira técnica com maiores taxas de incidência proporcionar uma natureza de má absorção; outros descrevem que não existem diferenças significativas entre as técnicas.

Corroborando com o autor supracitado Adams e outros colaboradores (2019) afirmam que o quadro colelitíase que se desenvolve após procedimentos bariátricos são decorrentes da rápida perda de peso e aumento excessivo da concentração de colesterol na bile, tornando o paciente mais susceptível a formação de cálculos biliares. Além disso, descreve que o RYGB promove diminuição da taxa de colecistoquinina e com isso favorece a estase biliar, outro ponto é a disfunção da motilidade da vesícula biliar e a ausência do seu esvaziamento decorrente da SG são fatores primordiais para formação do quadro.

Coupaye e outros colaboradores (2017) ao realizarem um estudo prospectivo em 2004 identificou que não ocorreu diferença na perda de peso em relação ao uso da técnica cirúrgica utilizada (RYGB ou SG) nos primeiros 6 meses de uso da UDCA. Ao comparar as doses utilizadas na prevenção do quadro de CL, foi identificado que a dose de $1000 \mathrm{~g} / \mathrm{dia}$ em pacientes pós RYGB apresentaram incidência menor (18,6\%); enquanto que pacientes pós SG na dose de $500 \mathrm{mg} /$ dia com uma taxa de 2,4\%, no qual essa diferença pode estar associada a natureza de absorção que o UDCA sofre a depender de cada procedimento. Entretanto, apesar de resultados positivos, o estudo apresenta como falha o número elevado de pacientes que desistiram durante o acompanhamento, com uma taxa de $40 \%$ no grupo placebo e $11 \%$ no grupo que estava sendo tratado. No estudo em questão foi identificado uma taxa de $16 \%$ e $10 \%$ em paciente que não fizeram uso do UDCA pós RYGB e SG respectivamente. Vale salientar, que o custo para utilização do tratamento medicamento apresenta muito mais inferior do que o uso do procedimento cirúrgico. 
Outro ponto é que a dose de 500mg/dia de UDCA por um período de 6 meses, além de promover um impacto significativo do quadro clinico do paciente após realização de cirurgia bariátrica, apresenta custo benefício significativo tanto para o paciente, quanto para o ambiente hospitalar ao fazer uma comparação quando é realizado uma colecistectomia (Della Penna et al., 2019).

Nabil, Khalil e Gamal (2019) realizaram um estudo prospectivo no qual ao comparar os pacientes que fizeram uso do UDCA na dose de $500 \mathrm{mg} / \mathrm{dia}$, com os que fizeram uso de placebo ocorreu o desenvolvimento de CL em $40 \%(\mathrm{P}<0,001)$ dos pacientes do grupo controle após realização gastrectomia vertical laparoscópica (LSG), com isso sendo possível avaliar que o uso da medicação pode ser considerada uma proposta adequada para diminuição da incidência da CL.

Della Penna e outros colaboradores (2019) realizaram um estudo retrospectivo com 61 pacientes que realizaram cirurgia bariátrica e que apresentava como diagnostico prévio um quadro de colelitíase assintomática - 63,9\% sendo submetidos à SG e 36,1\% a RYGB - e que realizaram um tratamento profilático secundário durante 6 meses após o procedimento cirúrgico com o UDCA, no qual foi possível avaliar que mesmo após uma perda significativa de peso que é considerado como fator crucial para o desenvolvimento de novos cálculos, identificou-se que apenas um paciente apresentou sintomatologia, demonstrando que seu uso apresenta impacto significativo na morbidades dos pacientes. Além disso, é notório que o uso profilático do UDCA em doses adequadas promove uma diminuição da formação de colelitíase, como também da necessidade de colecistectomia após a realização da cirurgia bariátrica. Vale ressaltar que após a retirada da medicação, apenas 2 pacientes apresentaram sintomáticos, e mesmo assim, não foi necessário a realização do colecistectomia.

Em um estudo retrospectivo realizado em pacientes com obesidade mórbida que foram submetidos a gastrectomia vertical laparoscópica (LSG) e fizeram uso do UDCA por um período de 6 meses foram acompanhados durante 12 meses verificou-se uma porcentagem de excesso de perda de peso (\% PEP) variando entre 71,5 $\pm 20,4$ no primeiro ano de pós-operatório, com uma incidência de CL de 0\% com 6 meses de pós operatório, como também após 12 meses. Além disso, percebe-se que a incidência geral de CL pósLSG é de aproximadamente de 2 a $5 \%$ no grupo que não fez uso da UCDA, que é semelhante a incidência de cálculos biliares sintomáticos que ocorrem após a LSG (3,8 e $7,5 \%$ ). Vale salientar, que os principais fatores de risco associados ao desenvolvimento de 
CL identificados foram a presença do quadro de dislipidemia, uma perda excessiva de peso após 3 meses do procedimento cirúrgico e a não utilização do UDCA (Abdallah et al., 2017)

Além disso, durante a análise dos estudos é notório que a administração do UDCA é segura, com poucos efeitos colaterais, com resultados que independem de fatores sociodemográficos. Por fim são perceptíveis que a adesão e tempo de uso são fatores cruciais para melhores resultados.

Adams e outros colaboradores (2019) afirmam que dados significativos que descrevem uma redução só foram identificadas $(\mathrm{p}=0,032)$ nos pacientes que apresentaram conformidade com a prescrição, sendo assim, essencial uma adesão adequada para que seja possível identificar uma resposta clinica eficaz. Vale ressaltar que os autores descrevem que um dos motivos para não adesão ao uso do UDCA é o próprio custo do seu uso.

Vala salientar que o uso do UDCA é identificado como uma modalidade terapêutica segura, que apresenta adesão adequada, efetividade e poucos efeitos colaterais, - os principais efeitos colaterais foram náuseas e vômitos. Além disso, promove uma redução da morbimortalidade, como a diminuição de complicações decorrente da obstrução biliar, como por exemplo, o quadro de pancreatite (Nabil; Khalil; Gamal, 2019).

\section{Considerações Finais}

Portanto a revisão sistemática foi composta por seis que avaliaram a eficiência e a segurança da administração de UDCA, com o objetivo de promover efeito profilático na formação de cálculos biliares após cirurgia bariátrica e consequentemente diminuir a incidência do CL. Esses estudos sugerem que a administração de 500 a 1000 mg de UDCA por um período de 6 meses, a depender da técnica utilizada esta significativamente associada a diminuição da incidência do quadro de CL, com boa adesão, custo-efetividade e poucos efeitos colaterais. Portanto, é notório que o seu apresenta resultados significativos e sendo essencial a realização de mais estudos que abordem a temática.

As pesquisas sobre o tema são recentes, sendo a mais longa efetuada num período de dez anos, no estudo é notório que existem resultados significativos na diminuição do desenvolvimento de quadro de cálculo biliar, entretanto, torna-se necessário a realização de mais estudos multicêntricos que descrevam o efeito do UDCA por um período mais prolongado. 
A falha metodológica mais observada nos estudos foi à ausência da formação de um grupo-controle em um dos estudos analisados, a falta de continuidade no acompanhamento dos pacientes em outro estudo e com isso podendo falsear os resultados identificados, como também o número pequeno de estudos incluídos na análise final, e com isso apresentando certa limitação.

\section{Referências}

Abdallah, Emad et al. (2017). Role of ursodeoxycholic acid in the prevention of gallstone formation after laparoscopic sleeve gastrectomy. Surgery today, v. 47, n. 7, p. 844-850;

Adams, Lindsay B. et al (2016). Randomized, prospective comparison of ursodeoxycholic acid for the prevention of gallstones after sleeve gastrectomy. Obesity surgery, v. 26, n. 5, p. 990-994;

Coupaye, Muriel et al (2017). Evaluation of incidence of cholelithiasis after bariatric surgery in subjects treated or not treated with ursodeoxycholic acid. Surgery for Obesity and Related Diseases, v. 13, n. 4, p. 681-685;

Della Penna, Andrea et al. (2019). Ursodeoxycholic Acid for 6 Months After Bariatric Surgery Is Impacting Gallstone Associated Morbidity in Patients with Preoperative Asymptomatic Gallstones. Obesity surgery, v. 29, n. 4, p. 1216-1221;

Ferrari, M. A. (2014). Colelitíase em pacientes bariátricos: correlação da perda de peso com a incidência de colelitíase em pacientes após a realização do bypass gastrintestinal. PUCRS Biblioteca Digital de Teses e Dissertações;

Machado, Francisco Heine Ferreira et al. (2019). Ursodeoxycholic acid in the prevention of gallstones in patients subjected to Roux-en-Y gastric bypass. Acta Cir Bras, v. 34, p. 1;

Nabil, Tamer M.; Khalil, Ahmed H.; Gamal, Kareem (2019). Effect of oral ursodeoxycholic acid on cholelithiasis following laparoscopic sleeve gastrectomy for morbid obesity. Surgery for Obesity and Related Diseases;

Sociedade Brasileira de Cirurgia Bariátrica e Metabólica (2018). Número de cirurgias bariátricas no Brasil aumenta 46,7\%. Disponivel em: <https://www.sbcbm.org.br/numerode-cirurgias-bariatricas-no-brasil-aumenta-467/>. Acesso em 08 de novembro de 2019.

\section{How to cite this article (APA format):}

Magalhães, Bráulio Filgueira; Leite, Pedro de Sousa; Moreira, Gabriella Rocha; Araújo, Yanny Brena Alencar; Cruz, Natália Mendes; Rolim, Francisco Felippe de Araújo. (2019). The use of Ursodeoxycholic Acid to prevent Cholelithiasis after Bariatric Surgery: A Systematic Literature Review. Am. In. Mult. J., October a December. (7) 4, 254-267. 
Received: 12/02/2019

Accepted: 14/12/2019 